\title{
Protective effect of Xuebijing injection against acute lung injury induced by left ventricular ischemia/reperfusion in rabbits
}

\author{
MINGLI JI ${ }^{1}$, YUXIA WANG ${ }^{2}$, LEI WANG ${ }^{3}$, IIPING CHEN $^{3}$ and $\mathrm{JING} \mathrm{LI}^{4}$ \\ Departments of ${ }^{1}$ Physiology and ${ }^{2}$ Pathophysiology, Xinxiang Medical University, Xinxiang, Henan 453003; \\ Departments of ${ }^{3}$ General Surgery and ${ }^{4}$ Otorhinolaryngology, The First Affiliated Hospital of Xinxiang Medical University, \\ Weihui, Henan 453100, P.R. China
}

Received January 14, 2015; Accepted February 19, 2016

DOI: $10.3892 /$ etm.2016.3275

\begin{abstract}
Xuebijing (XBJ) is a Chinese herbal preparation. Previous studies have demonstrated that XBJ injection is able to inhibit the uncontrolled release of endogenous inflammatory mediators, attenuate inflammation, and alleviate organ damage. However, there are no relevant reports on the protective effect of XBJ against left ventricular ischemia/reperfusion (I/R)-induced acute lung injury (ALI). Therefore, the aim of the present study was to evaluate the protective effect of XBJ on ALI induced by left ventricular $\mathrm{I} / \mathrm{R}$, and provide evidence for the clinical application of XBJ. In the present study, 120 healthy rabbits of mixed gender were randomly assigned to a normal control group, ischemia group, I/R group (I/RG) and XBJ-injection treatment group (TG). In addition, each group was further divided into three subgroups ( $n=10 /$ subgroup), namely, $30 \mathrm{~min}$ pre-ischemia, $30 \mathrm{~min}$ post-ischemia and $30 \mathrm{~min}$ post-reperfusion subgroups. Blood samples $(5 \mathrm{ml})$ were collected from the jugularis externa and carotis communis of the rabbits at the three time points, and a blood gas analyzer was used to measure the arterial partial pressure of oxygen $\left(\mathrm{PaO}_{2}\right)$ and carbon dioxide $\left(\mathrm{PaCO}_{2}\right)$. Following sacrifice, the lungs of the rabbits were removed and a bronchoalveolar lavage (BAL) was
\end{abstract}

Correspondence to: Professor Mingli Ji, Department of Physiology, Xinxiang Medical University, 601 East of Jinsui Road, Xinxiang, Henan 453003, P.R. China

E-mail: teachermingliji@163.com

Abbreviations: ICAM-1, intercellular adhesion molecule-1; TNF- $\alpha$, tumor necrosis factor- $\alpha$; XBJ, Xuebijing injection; $C G$, control group; IG, ischemia group; I/RG, ischemia/reperfusion group; TG, treatment group; T1, 30 min pre-ischemia; T2, 30 min post-ischemia; T3, 30 min post-reperfusion; $\mathrm{PaO}_{2}$, partial pressure of oxygen; $\mathrm{PaCO}_{2}$, partial pressure of carbon dioxide; BALF, bronchoalveolar lavage fluid; ELISA, enzyme-linked immunosorbent assay; ECG, electrocardiogram; ALI, acute lung injury

Key words: Xuebijing injection, acute lung injury, left ventricular ischemia reperfusion, intercellular adhesion molecule-1, tumor necrosis factor- $\alpha$ immediately performed. An enzyme-linked immunosorbent assay was used to measure the expression levels of tumor necrosis factor- $\alpha$ (TNF- $\alpha$ ) in the BAL fluid (BALF) and peripheral blood. In addition, the lower lobe of the right lung was removed in order to measure the protein expression levels of intercellular adhesion molecule-1 (ICAM-1) and TNF- $\alpha$. The results demonstrated that in the rabbits of the TG $\mathrm{PaO}_{2}$ was increased, $\mathrm{PaCO}_{2}$ was decreased, the lung tissue congestion edema was attenuated, the expression levels of TNF- $\alpha$ in the peripheral blood and BALF were reduced and the protein expression levels of ICAM-1 and TNF- $\alpha$ in the lung tissue samples were decreased, as compared with those in the I/RG rabbits. These results suggest that XBJ may protect against left ventricular I/R-induced ALI by regulating the expression of the inflammatory mediators TNF- $\alpha$ and ICAM-1.

\section{Introduction}

Acute myocardial infarction is a common medical emergency of the cardiovascular system. The clinical treatments for myocardial infarction mainly focus on interventional or thrombolytic therapy. Although interventional or thrombolytic therapy is able to clear the coronary artery and restore blood flow (1), it can also cause reperfusion injury (2). This means that blood reperfusion may occasionally further aggravate the damage (3), and can even cause respiratory dysfunction, with patients with severe cases succumbing to respiratory failure (4). Therefore, the development of drugs that effectively control or reduce ischemia/reperfusion (I/R)-induced lung injury is required. A previous study has found that an imbalance of inflammatory mediators is an important cause of myocardial I/R injury (5). Thus, redressing the balance of inflammatory mediators is an important target for reducing or preventing I/R injury.

Xuebijing $(\mathrm{XBJ})$ is a traditional Chinese medicine preparation (6). In clinical settings, XBJ is combined with antibiotics to treat severe acute lung diseases, including acute lung injury (ALI) (7), sepsis (8) and multiple organ dysfunction syndrome (9). However, to the best of our knowledge, no previous studies have investigated the protective effects of XBJ injection against left ventricular I/R-induced ALI. Therefore, the present study established a rabbit model of left ventricular I/R-induced ALI in order to investigate the underlying pathogenic mechanisms and the protective role of $\mathrm{XBJ}$ on respiratory 
function. The following parameters were assessed: The partial pressure of oxygen $\left(\mathrm{PaO}_{2}\right)$ and carbon dioxide $\left(\mathrm{PaCO}_{2}\right)$; the expression levels of tumor necrosis factor (TNF)- $\alpha$ in peripheral blood and bronchoalveolar lavage (BAL) fluid (BALF); the lung wet/dry weight ratio (W/D); and the protein expression levels of intercellular adhesion molecule-1 (ICAM-1) and TNF- $\alpha$ in the rabbit lung tissue samples.

\section{Materials and methods}

Animals and grouping. A total of 120 healthy adult (6-7 months old) mixed-gender New Zealand rabbits weighing 200-250 g were obtained from the Experimental Animal Center of Zhengzhou University (Zhengzhou, China). Each rabbit was housed in an individual cage (at $20 \pm 5^{\circ} \mathrm{C}$ ) under a 12:12 h light and dark cycle for 1 week prior to experimentation. All rabbits had free access to water, and food was removed $8 \mathrm{~h}$ prior to the study. The rabbits were randomly divided into four groups

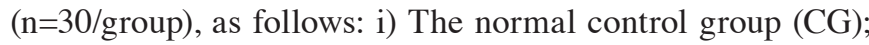
ii) the ischemia group (IG); iii) the I/R group (I/RG); and iv) the $\mathrm{I} / \mathrm{R}+\mathrm{XBJ}$ treatment group (TG). In addition, each group was further divided into three subgroups ( $n=10 /$ subgroup) as follows: i) $30 \mathrm{~min}$ pre-ischemia (T1); ii) $30 \mathrm{~min}$ post-ischemia (T2); and iii) $30 \mathrm{~m}$ in post-reperfusion (T3). For $12 \mathrm{~h}$ prior to the experiment, the rabbits were fasted but had ad libitum access to water. All experimental procedures were approved by the Ethics Committee of Xinxiang Medical University (Xinxiang, China). The present study was conducted in accordance with internationally recognized guidelines on animal welfare (10), as well as local and national regulations.

Reagents and instruments. The instruments included a Leica RM2235 microtome (Leica Microsystems GmbH, Wetzlar, Germany), a CR-21G High-Speed Refrigerated Centrifuge (Hitachi, Ltd., Tokyo, Japan), a DG5033A Enzyme-linked Immunosorbent Assay (ELISA) Analyzer (Shanghai Bogoo Biotechnology, Co., Ltd., Shanghai, China), a fully automated M248 Blood Gas Analyzer (Bayer China Ltd., Shanghai, China) and a BL-420 Biological Signal Collecting and Processing system (Chengdu TME Technology, Co., Ltd., Chengdu, China). The main reagents were as follows: XBJ (10 ml ampoules; Tianjin Chase Sun Pharmaceutical, Co., Ltd., Tianjin, China), 20\% urethane (Shanghai Yunqiang Chemical, Co., Ltd., Shanghai, China), and ICAM-1 and TNF- $\alpha$ polyclonal antibodies (Beijing Biosynthesis Biotechnology Co., Ltd., Beijing, China). Surgical instruments and general laboratory supplies were provided by the Functional Laboratory of Xinxiang Medical University.

Surgical procedure. The rabbits underwent general anesthesia with $20 \%$ urethane $(5 \mathrm{ml} / \mathrm{kg})$ via the ear vein, followed by isolation and intubation of the trachea, right jugularis externa and left carotis communis. Following a thoracotomy, the left anterior descending coronary artery was separated and ligated, with the ST segment elevation arched upward, and with regional myocardial darkening serving as an effective indicator of ischemia. In the CG, the left anterior descending coronary artery was separated but not ligated, and the rabbits were observed for $4 \mathrm{~h}$. In the IG, myocardial ischemia was established by making a slipknot using a 6-0 silk suture around the left anterior descending coronary artery for $1.5 \mathrm{~h}$. In the I/RG, following ligation of the left anterior descending coronary artery for $30 \mathrm{~min}$, the ligature was loosened and the blood supply was restored. The TG underwent the same procedures as the I/RG, and were also given XBJ $(10 \mathrm{ml} / \mathrm{kg})$ at two time points: $10 \mathrm{~min}$ prior to ischemia and immediately following reperfusion. Prior to the thoracotomy, needle electrodes were inserted into the limbs and chest, and connected to the BL-420 Biological Signal Collecting and Processing system. The electrocardiogram (ECG; paper speed, $50 \mathrm{~mm} / \mathrm{sec}$; gain, $1 \mathrm{mV}=20 \mathrm{ram}$ ) and diaphragm discharge curve were monitored continuously. One end of a lead was connected to a hook positioned on the skin at the point of strongest respiratory movement, and the other end of the lead was connected to a pressure transducer chip connected to the BL-420 system for the recording of respiratory curves.

Samples. At the T1, T2 and T3 time points, 5-ml peripheral blood samples were collected from the left common carotid artery, and the $\mathrm{PaO}_{2}$ and $\mathrm{PaCO}_{2}$ were measured using the M248 Blood Gas Analyzer. Also, 2-ml venous blood samples were collected from the right external jugular vein for measurement of the level of TNF- $\alpha$. Subsequently, all rabbits were sacrificed by aeroembolism and the lungs were harvested. BALF samples $(5 \mathrm{ml})$ were collected using Y-type tracheal intubation with one inlet connected to a 50-ml syringe of air, and another connected a $10-\mathrm{ml}$ syringe containing $10 \mathrm{ml} 0.9 \% \mathrm{NaCl}$. Air and $0.9 \% \mathrm{NaCl}$ were concurrently introduced into the lungs, and then the 10-ml syringe was pumped back and forth several times to collect the BALF. The lower lobe of the right lung was removed, fixed with paraformaldehyde solution $(1 \mathrm{ml} / 100 \mathrm{~g}$; Qingzhou Hengxing Chemical Co., Ltd., Qingzhou, China), embedded in paraffin (Dekang Medical Instrument Co., Ltd., Xinxiang, China) and cut into 5- $\mu \mathrm{m}$ sections using the Leica RM2235 microtome, prior to being fixed to glass slides coated with (3-aminopropyl)triethoxysilane (Dekang Medical Instrument Co., Ltd.).

ELISA. The level of TNF- $\alpha$ in the venous blood and BALF was measured by a double-antigen sandwich ELISA (EIA-1126; Beijing Zhongshan Golden Bridge Biological Technology, Co., Ltd.), according to the manufacturer's protocol. Briefly, each sample (sera; diluted with coating buffer) was applied in triplicate to 96 -well plates pre-coated with anti-TNF- $\alpha$ antibody and incubated overnight at $4^{\circ} \mathrm{C}$. Following this, the plates were washed three times with phosphate-buffered saline (PBS) and then incubated with biotinylated goat anti-rabbit IgG $(1: 20,000)$ for $2 \mathrm{~h}$ at $37^{\circ} \mathrm{C}$. The plates were then incubated with $0.01 \%$ 3,3',5,5'-tetramethylbenzidine (Beijing CellChip Biotechnology Co., Ltd., Beijing, China), after which $2 \mathrm{M}$ $\mathrm{H}_{2} \mathrm{SO}_{4}$ (Nanjing Taiye Chemical Industry Co., Ltd., Nanjing, China) was added to terminate the reaction. The optical density was recorded at $450 \mathrm{~nm}$ using a DG5033A ELISA Analyzer. The experiments were performed in triplicate. The sensitivity and specificity of the ELISA were 99 and 92\%, respectively.

Lung weight and water content. Lungs were weighed, and then dried in a vacuum drying oven (cat. no. DHG-9420; Shanghai Haixiang Equipment Factory, Shanghai, China) at $80^{\circ} \mathrm{C}$ for $48 \mathrm{~h}$ to obtain the dry weight. The lung wet 

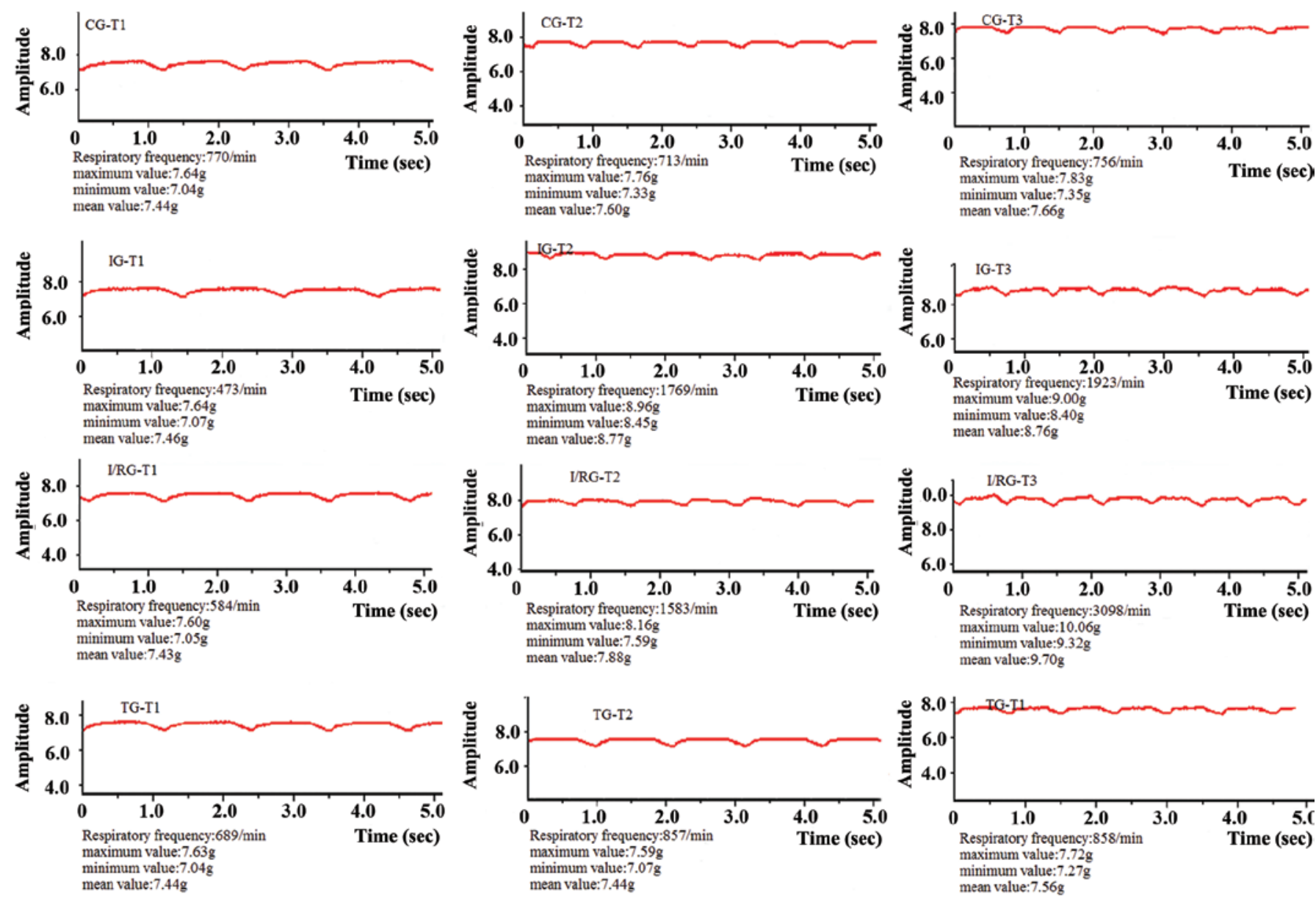

Figure 1. Comparison of the respiratory curves of the CG, IG, I/RG and TG. CG, normal control group; IG, ischemia group; I/RG, ischemia/reperfusion group; TG, treatment group; T1, 30 min pre-ischemia; T2, 30 min post-ischemia; T3, 30 min post-reperfusion.

weight/dry weight (W/D) ratios of left lung tissue samples were determined, and the water content of the lung tissue was calculated using the following formula: Water content $(\%)=($ wet weight - dry weight $) /$ wet weight $x 100$.

Immunohistochemistry. Lung tissue sections, fixed in formalin (Shanghai Fengshou Biotechnology Co., Ltd., Shanghai, China) and embedded in paraffin, were deparaffinized, routinely rehydrated using graded ethanol, peroxidase-quenched with $3 \% \mathrm{H}_{2} \mathrm{O}_{2}$, blocked with 5\% normal goat serum (Amyjet Scientific, Inc., Wuhan, China) and probed with rabbit anti-rabbit ICAM-1 antibody (final concentration:0.1\%; bs-0608R) or rabbit anti-rabbit TNF- $\alpha$ antibody (final concentration:0.1\%; bs-2081R) overnight at $4^{\circ} \mathrm{C}$. The sections were then thoroughly washed with PBS for $5 \mathrm{~min}$, repeated three times. Subsequently, the tissue sections were incubated with goat anti-rabbit IgG secondary antibody (bs-0295Gs; Beijing Biosynthesis Biotechnology Co., Ltd.) at room temperature for $1 \mathrm{~h}$. The sections were thoroughly washed with PBS for $5 \mathrm{~min}$, repeated three times. Following this, sections were incubated with horseradish peroxidase (HRP)-conjugated rabbit anti-goat antibody (bs-0294R-HRP; Beijing Biosynthesis Biotechnology Co., Ltd.) at $4^{\circ} \mathrm{C}$ for $30 \mathrm{~min}$. Eventually, reaction products were visualized following diaminobenzidine (DAB) and hematoxylin (Shanghai Yantuo Biotechnology Co., Ltd., Shanghai, China) counterstaining.
The protein expression levels of ICAM-1 and TNF- $\alpha$ in the lung tissue samples of the rabbits at each of the T1, T2 and T3 time points were measured. These experiments were performed in triplicate. According to the results of a semi-quantitative method (11), 10 random fields were selected under a Nikon microscope (80i; Nikon Instruments Co., Ltd, Shanghai China), in which 100 cells were counted. The tissue sections were scored as follows: No staining, '-'; <25\% positive cells, '+'; $25-75 \%$ positive cells, '++'; and $>75 \%$ positive cells, '+++'.

Statistical analysis. Statistical analyses were conducted using SPSS software, version 11.0 (SPSS, Inc., Chicago, IL, USA). Data are presented as the mean \pm standard deviation. The data were analyzed using an Independent Samples t-test and categorical data were analyzed using a $\chi^{2}$ test. $\mathrm{P}<0.05$ was considered to indicate a statistically significant difference.

\section{Results}

Comparison of respiratory curves. Respiratory curves (Fig. 1) were collected and recorded using the BL-420 Biological Signal Collecting and Processing system. At the T1, T2 and T3 time points, the breathing of the rabbits in the CG was steady and the respiratory amplitude and duration did not significantly 
A

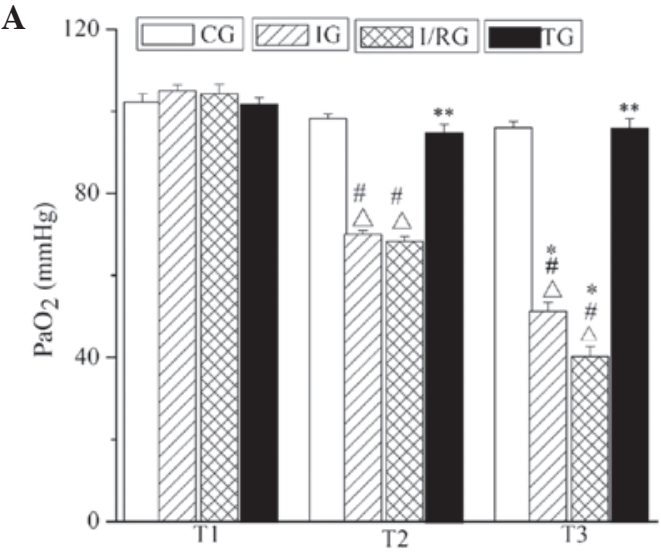

B

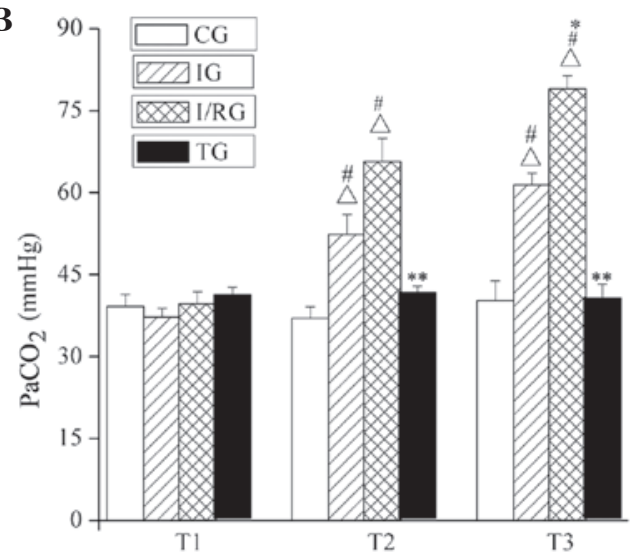

Figure 2. Comparison of $\mathrm{PaO}_{2}$ and $\mathrm{PaCO}_{2}$ among the groups at the T1, T2 and $\mathrm{T} 3$ time points. The (A) $\mathrm{PaO}_{2}$ and (B) $\mathrm{PaCO}$ in the CG, IG, I/RG and TG groups Data are presented as the mean \pm standard deviation. ${ }^{\Delta} \mathrm{P}<0.001$ vs. the $\mathrm{CG}$ at the same time; ${ }^{*} \mathrm{P}<0.001$ vs. T1 in the same group; ${ }^{*} \mathrm{P}<0.001$ vs. T2 in the same group; ${ }^{* *} \mathrm{P}<0.001$ vs. the IG and I/RG at the same time. CG, normal-control group; IG, ischemia group; I/RG, ischemia/reperfusion group; TG, treatment group; T1, 30 min pre-ischemia; T2, 30 min post-ischemia; T3, 30 min post-reperfusion; $\mathrm{PaO}_{2}$, partial pressure of oxygen; $\mathrm{PaCO}_{2}$, partial pressure of carbon dioxide.

A

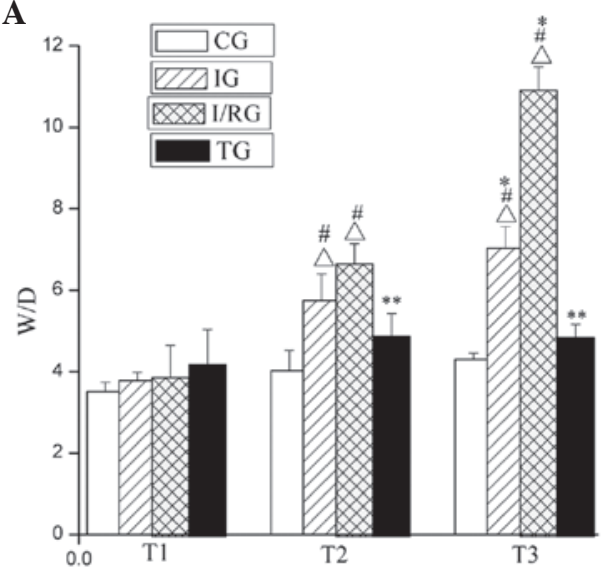

B

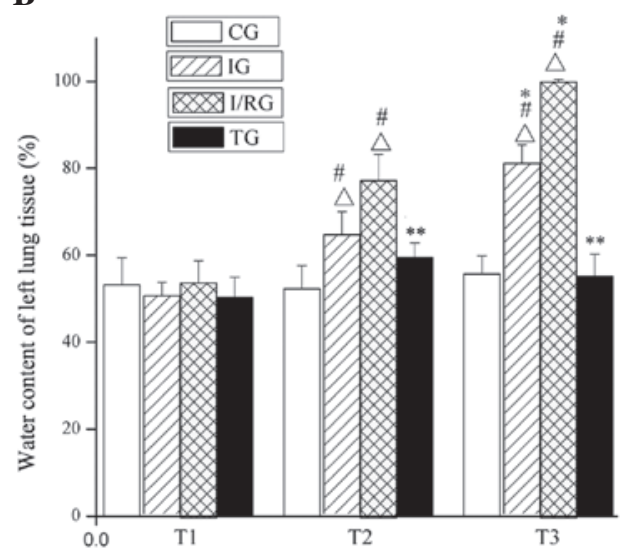

Figure 3. Comparison of the W/D and water content of left lung tissue from rabbits in the the four groups at the T1, T2 and T3 time points. The (A) W/D and (B) water content of the left lung tissue from rabbits in the CG, IG, I/RG and TG. Data are presented as the mean \pm standard deviation. ${ }^{\Delta} \mathrm{P}<0.001$ vs. the $\mathrm{CG}$ at the same time; ${ }^{*} \mathrm{P}<0.001$ vs. T1 in the same group; ${ }^{\mathrm{P}}<0.001 \mathrm{vs}$. T2 in the same group; ${ }^{* *} \mathrm{P}<0.001 \mathrm{vs}$. the IG and I/RG at the same time. CG, normal-control group; IG, ischemia group; I/RG, ischemia/reperfusion group; TG, treatment group; T1, 30 min pre-ischemia; T2, 30 min post-ischemia; T3, 30 min post-reperfusion; $\mathrm{W} / \mathrm{D}$, wet/dry weight ratio.

fluctuate. Conversely, the respiratory amplitude for the rabbits in the IG was shallow and the respiratory duration was short. In addition, respiratory fluctuations were observed between the three time points. In comparison with the IG, the respiratory amplitude for the rabbits in the I/RG was more shallow and the respiratory duration was shorter. The respiratory curves for the rabbits in the $\mathrm{TG}$ resembled those of the rabbits in the CG (Fig. 1).

Comparison of $\mathrm{PaO}_{2}$ and $\mathrm{PaCO}$. The $\mathrm{PaO}_{2}$ and $\mathrm{PaCO}_{2}$ were measured using the fully automated M248 Blood Gas Analyzer. The $\mathrm{PaO}_{2}$ and $\mathrm{PaCO}_{2}$ of rabbits in the TG were not significantly different from those in the $\mathrm{CG}$ at the $\mathrm{T} 1, \mathrm{~T} 2$ and T3 time points $(\mathrm{P}>0.05)$. The $\mathrm{PaO}_{2}$ values of the rabbits in the IG and I/RG were significantly decreased compared with those in the CG, at the T2 and T3 time points $(\mathrm{P}<0.001)$, and particularly decreased at T3 in the I/RG. Furthermore, the $\mathrm{PaCO}_{2}$ values of the rabbits in the IG and I/RG were significantly increased compared with those in the $\mathrm{CG}$, at the T2 and T3 time points ( $\mathrm{P}<0.001$; Fig. 2).
Comparison of W/D value and water content in the left lung tissue samples of the rabbits. At the T1, T2 and T3 time points, the W/D weight ratio and water content of the left lung tissue from the $\mathrm{CG}$ did not significantly differ $(\mathrm{P}>0.05)$. Conversely, those of the tissue from the IG and I/RG were significantly increased $(\mathrm{P}<0.001)$ compared with those in the $\mathrm{CG}$, with the greatest increases being observed for the I/RG. The W/D weight ratio and water content of the left lung tissue from the TG were not significantly different from those in the CG at any of the three time points ( $\mathrm{P}>0.05$; Fig. 3 ).

General observation of the rabbit lungs at T3. At the T3 time point, the lungs of rabbits in the CG were light pink and small in size. In the IG, the lungs appeared dark red and small, with an uneven surface and visible hemorrhages. Similarly, congestion and pulmonary edema were apparent in the lungs of the $\mathrm{I} / \mathrm{RG}$, and the volume was larger, the margins of the lungs were blunt, the color was uneven and clear spot bleeding and bruising was observed in the lower lobe of the lungs. In addition, white foamy/bloody liquid was overflowing from the lung 

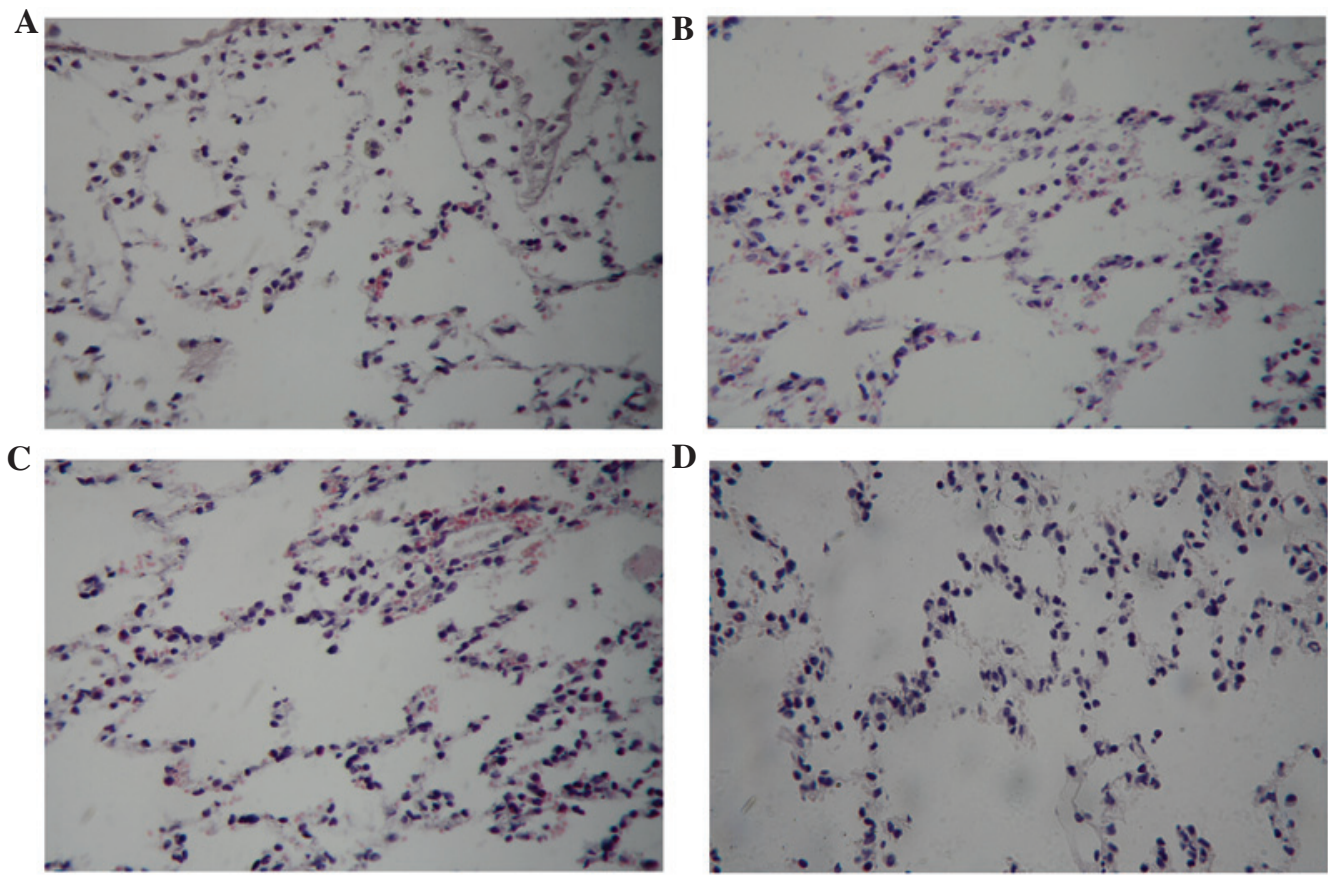

Figure 4. Light microscopy observation of the lung tissue samples of rabbits in the (A) normal control, (B) ischemia, (C) ischemia/reperfusion and (D) treatment groups at 30 min post-reperfusion following immunohistochemical (diaminobenzidine) staining. Magnification, $\mathrm{x} 400$.

section. Conversely, the TG lungs exhibited only mild edema, appeared red in color and had a smooth surface.

Light microscopy observations of the rabbit lungs at T3. At the T3 time point, the alveolar structure of the lungs in the CG group was clear, the walls of the alveolae were thin and no inflammatory cells had infiltrated into the alveolar space and lung interstitium. In the IG, pulmonary vascular congestion, focal alveolar hemorrhages and small focal alveolar collapse/atelectasis were observed in some areas of the lung. In the I/RG, dilatation and congestion was observed in the pulmonary capillaries, the alveolar septum was thickened, a large number of leukocytes had infiltrated and aggregated and hemorrhage and exudation were detected in the alveolar cavity. In addition, atelectasis was observed in some alveolae. Conversely, in the TG, the alveolar structure was relatively complete and hemorrhage and exudation of the alveolar was markedly reduced, as compared with the I/RG lungs, although alveolar atrophy and infiltrated inflammatory cells were infrequently observed (Fig. 4).

Expression levels of $T N F-\alpha$ in the BALF and the peripheral blood. The expression levels of TNF- $\alpha$ in the BALF and peripheral blood from the four groups were detected by performing an ELISA. At the T1, T2 and T3 time points, there was no significant difference in the expression levels of TNF- $\alpha$ in the peripheral blood and BALF between the CG and TG $(\mathrm{P}>0.05)$. In the IG, the expression levels of TNF- $\alpha$ in the peripheral blood and BALF were increased at T2, and reached a maximum at $\mathrm{T} 3(\mathrm{P}<0.001)$, these changes were more pronounced in the I/RG $(\mathrm{P}<0.001)$. At $\mathrm{T} 2$ and $\mathrm{T} 3$, the expression levels of TNF- $\alpha$ in the peripheral blood and BALF in the TG were significantly lower compared with those in the IG and I/RG (P<0.001; Fig. 5).
Protein expression of ICAM-1 and TNF- $\alpha$ in the rabbit lung tissue samples at T3. The immunoreactive product of ICAM-1 was brown and was observed in the membrane and cytoplasm. TNF- $\alpha$ formed dark brown-to-yellow granules that were observed in the cytoplasm. As shown Figs. 6 and 7, there was an evident increasing trend in the expression of ICAM-1 and TNF- $\alpha$ from CG and IG to I/RG at T3, and the levels of ICAM-1 and TNF- $\alpha$ in the TG were comparable to those in the CG and markedly lower than those in the IG and I/RG.

Comparison of the association between the protein expression levels of ICAM-1 and TNF- $\alpha$ in the rabbit lung tissue samples at T3. The association between the protein expression levels of ICAM-1 and TNF- $\alpha$ in the rabbit lung tissues samples at T3 was investigated. A positive correlation between the protein expression levels of ICAM-1 and TNF- $\alpha$ was observed (Table I).

\section{Discussion}

Due to the structural characteristics of the left anterior descending coronary artery, the blood supply of the heart is prone to ischemia (12-14), and the recovery of blood perfusion to the ischemic myocardium is an important therapeutic strategy that reduces the occurrence of ischemic injury. However, in some cases reperfusion may further aggravate damage or induce irreversible damage; this phenomenon is called reperfusion injury. As an important organ with roles in immunity and metabolism, the lung has a high probability of being affected by reperfusion injury and is prone to inflammation (15).

Previous studies have demonstrated that the activation of a large number of neutrophils occurs in the early stages of ischemia (16), and that these neutrophils enhance the process of reperfusion (17). In addition, activated neutrophils promote the 
A

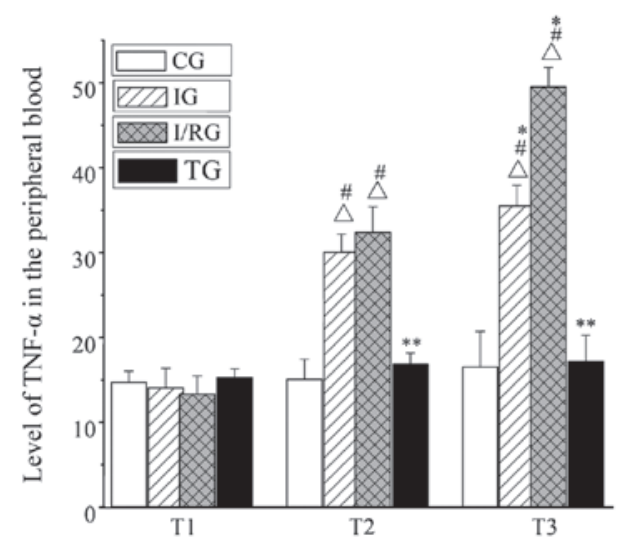

B

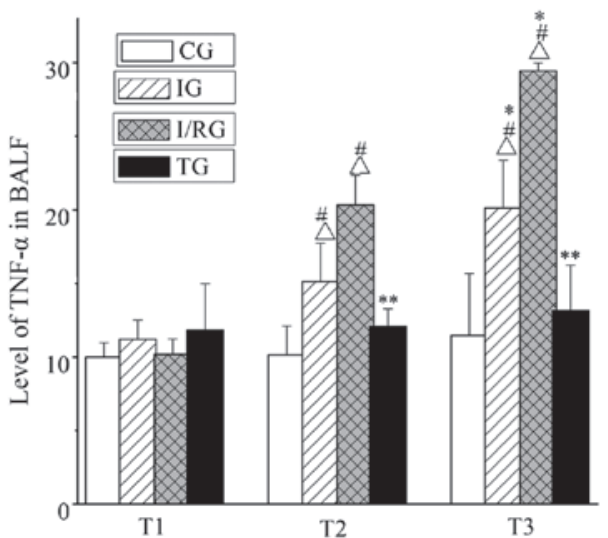

Figure 5. Expression levels of TNF- $\alpha$ in the peripheral blood and BALF in the four groups at the T1, T2 and T3 time points. The expression levels of TNF- $\alpha$ in the (A) peripheral blood and (B) BALF. Data are presented as the mean \pm standard deviation. ${ }^{\Delta} \mathrm{P}<0.001$, at the same time vs. the CG; ${ }^{\#} \mathrm{P}<0.001$, the same group vs. T1; "P $<0.001$, the same group vs. T2; *" $\mathrm{P}<0.001$, at the same time vs. the IG and I/RG. CG, normal-control group; IG, ischemia group; I/RG, ischemia/reperfusion group; TG, treatment group; T1, 30 min pre-ischemia; T2, 30 min post-ischemia; T3, 30 min post-reperfusion; BALF, brochoalveolar lavage fluid.

A

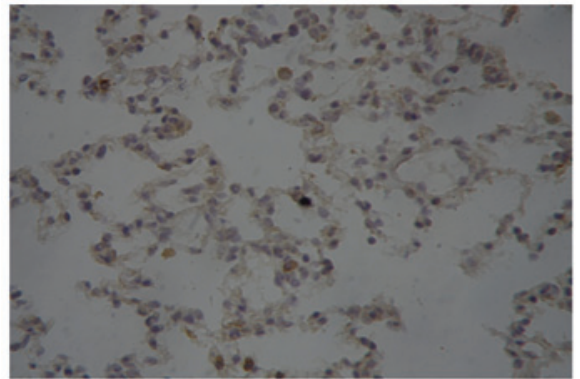

B

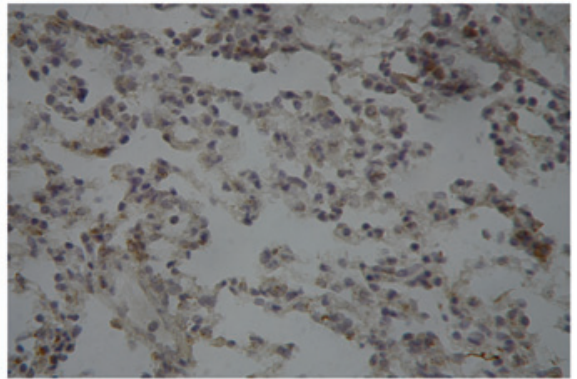

C

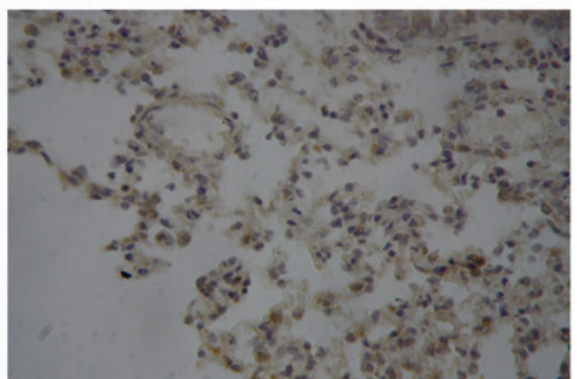

D

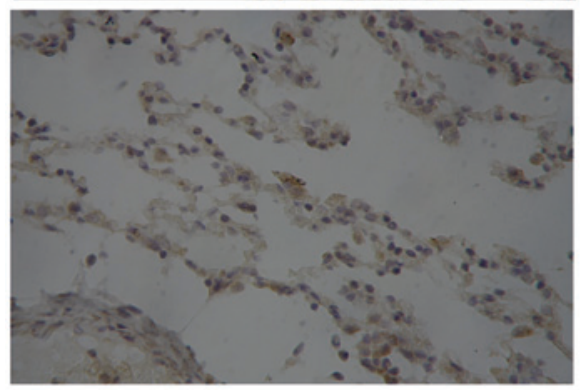

Figure 6. Expression of intercellular adhesion molecule-1 in lung tissue samples of rabbits in the (A) normal-control, (B) ischemia, (C) ischemia/reperfusion and (D) treatment groups 30 min post-reperfusion following immunohistochemical (diaminobenzidine) staining. Magnification, x400.
A

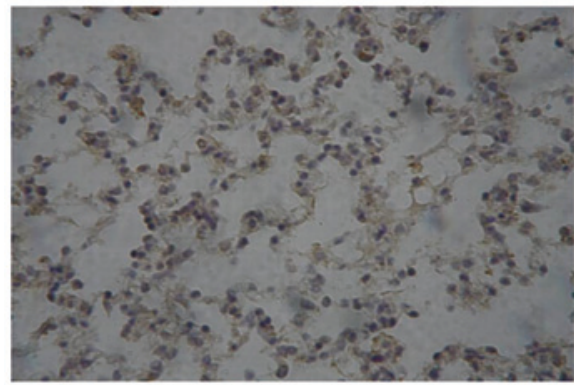

B

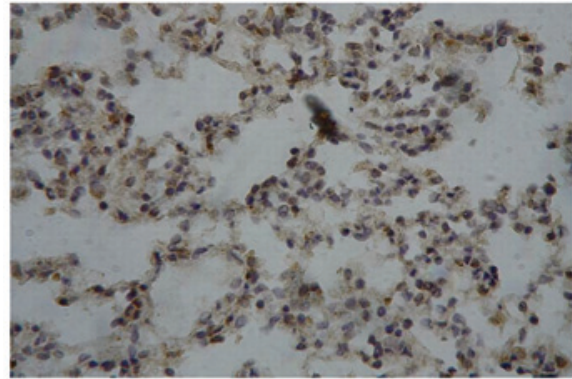

C

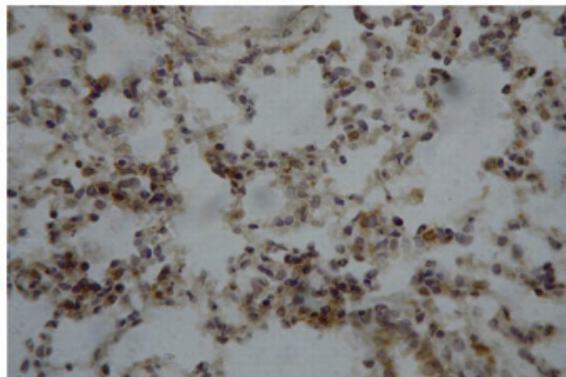

D

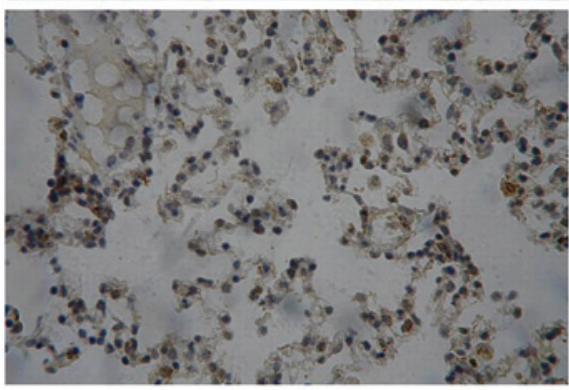

Figure 7. Protein expression of tumor necrosis factor- $\alpha$ in lung tissue samples of rabbits in the (A) normal-control, (B) ischemia, (C) ischemia/reperfusion and (D) treatment groups 30 min post-reperfusion following immunohistochemical (diaminobenzidine) staining. Magnification, x400. 
Table I. Comparison of the association between the protein expression levels of ICAM-1 and TNF- $\alpha$ in the lung tissue samples of rabbits in the 4 groups at the T3 time point.

\begin{tabular}{|c|c|c|c|c|}
\hline \multirow[b]{2}{*}{ TNF- $\alpha$} & \multicolumn{4}{|c|}{ ICAM-1 } \\
\hline & - & + & ++ & +++ \\
\hline \multicolumn{5}{|l|}{ CG } \\
\hline- & 6 & 1 & 0 & 0 \\
\hline+ & 1 & 2 & 0 & 0 \\
\hline++ & 0 & 0 & 0 & 0 \\
\hline+++ & 0 & 0 & 0 & 0 \\
\hline \multicolumn{5}{|l|}{ IG } \\
\hline- & 2 & 1 & 0 & 0 \\
\hline+ & 0 & 3 & 1 & 0 \\
\hline++ & 0 & 2 & 0 & 0 \\
\hline+++ & 0 & 0 & 1 & 0 \\
\hline \multicolumn{5}{|l|}{$\mathrm{I} / \mathrm{RG}$} \\
\hline- & 0 & 1 & 0 & 0 \\
\hline+ & 0 & 3 & 2 & 0 \\
\hline++ & 0 & 1 & 0 & 0 \\
\hline+++ & 0 & 0 & 2 & 1 \\
\hline \multicolumn{5}{|l|}{$\mathrm{TG}$} \\
\hline- & 4 & 1 & 1 & 0 \\
\hline+ & 1 & 2 & 0 & 0 \\
\hline++ & 0 & 1 & 0 & 0 \\
\hline+++ & 0 & 0 & 0 & 0 \\
\hline
\end{tabular}

ICAM-1, intercellular adhesion molecule-1; TNF- $\alpha$, tumor necrosis factor- $\alpha$; CG, normal-control group; IG, ischemia group; $\mathrm{I} / \mathrm{RG}$, ischemia/reperfusion group; TG, treatment group; T3, $30 \mathrm{~min}$ post-reperfusion; '-', no positive cells; '+', $<25 \%$ positive cells; '++', $25-75 \%$ positive cells; ' +++ ', $>75 \%$ positive cells.

release of large amounts of inflammatory cytokines, including TNF- $\alpha(18,19)$, interleukin (IL)-6 (20,21), IL-10 (22-24) and ICAM-1 (25), in order to expand the inflammatory response. ICAM-1 has a key role in neutrophil aggregation, activation and the release of inflammatory mediators (26-28). Under normal circumstances, the protein expression levels of ICAM-1 are negligible, and this prevents pathological damage to the body (29). However, during I/R and other pathological conditions, the expression of proinflammatory mediators is stimulated and the expression levels of ICAM-1 are increased significantly (30). This in turn promotes the adherence of neutrophils to endothelial cells and their migration across the endothelial barrier, resulting in inflammation and various pathological changes that characterize reperfusion injury (7).

$\mathrm{XBJ}$ is a traditional Chinese medicine preparation with anti-bacterial, anti-endotoxin and anti-inflammatory properties (31). XBJ is a herbal preparation consisting mainly of red peony root, chuanxiong, Salvia divinorum and Angelica sinensis. XBJ has a critical role in inhibiting the generation of free oxygen radicals and endotoxins, as well as the uncontrolled release of inflammatory mediators (32).
The present study established a rabbit model of left ventricular I/R-induced ALI, in order to investigate the protective effects of XBJ against lung reperfusion injury. The results of the present study demonstrated that, as compared with the CG and IG, the respiratory amplitude of rabbits in the I/RG was shallow, the respiratory frequency was increased, the $\mathrm{PaO}_{2}$ and $\mathrm{PaCO}_{2}$ were decreased and the lung volume was enlarged at the T3 time point, thus suggesting that ALI occurred in the rabbits of the I/RG. Conversely, for the rabbits in the TG, the $\mathrm{PaO}_{2}$ and $\mathrm{PaCO}_{2}$ increased, edema and hyperemia of the lung tissue was reduced and the W/D weight ratio was decreased, suggesting that XBJ was able to improve respiratory function. A quantitative analysis revealed that, as compared with that in the CG, the levels of TNF- $\alpha$ in the peripheral blood and BALF were significantly increased in the IG at T2, and reached a peak at T3. Similarly, the TNF- $\alpha$ levels were significantly increased in the I/RG at T2 and T3, as compared with the $\mathrm{CG}$, and at T3, as compared with IG. Conversely, there was no significant difference between CG and TG at the T1, T2 and T3 time points.

A gross observation of the lung tissue indicated that lung tissue injury occurred in the IG, and was aggravated in the I/RG at T3. Conversely, lung tissue damage in the TG was markedly attenuated, as compared with that in the I/RG. Immunohistochemical analyses demonstrated that the expression levels of ICAM-1 and TNF- $\alpha$ exhibited an increasing trend from $\mathrm{CG}$ and $\mathrm{IG}$ to $\mathrm{I} / \mathrm{RG}$ at $\mathrm{T} 3$, whereas the expression levels of ICAM-1 and TNF- $\alpha$ in the TG were markedly lower, as compared with the IG and I/RG. A correlation analysis demonstrated that the expression levels of ICAM-1 were positively correlated with the expression levels of TNF- $\alpha$ in the IG, I/RG and TG at T3. These results suggested that, during the pathogenesis of left ventricular I/R-induced ALI, a large number of neutrophils were activated, promoting the release of proinflammatory mediators, including TNF- $\alpha$. The excessive release of TNF- $\alpha$ in turn induced the overexpression of ICAM-1, which led to the adhesion of neutrophils to endothelial cells, eventually leading to lung inflammatory injury. XBJ exhibited a protective effect in the rabbit lung tissue samples, inhibited the excessive release of early inflammatory cytokines, including $\mathrm{TNF}-\alpha$, and decreased the expression levels of ICAM-1.

In conclusion, the present study investigated the effect of $\mathrm{XBJ}$ on left ventricular I/R-induced ALI by performing a blood gas analysis and measuring the expression levels of ICAM-1 and TNF- $\alpha$. The results demonstrated that, following treatment with XBJ, the release of the inflammatory mediators ICAM-1 and TNF- $\alpha$ was effectively inhibited, and the $\mathrm{PaO}_{2}$ was improved. Understanding the molecular mechanism underlying the anti-inflammatory effect of XBJ will provide a theoretical basis and experimental evidence for its clinical application.

\section{Acknowledgements}

The present study was supported by grants from the Foundation of Henan Educational Commission (grant no. 2011GGJS-127) and the Henan Science and Technology Bureau (grant no. 132300410160). The authors would like to thank Professor Zhibin Qian for providing the facilities to carry out the study. 


\section{References}

1. Waldo SW, Brenner DA, Li S, Alexander K and Ganz P: Reperfusion times and in-hospital outcomes among patients with an isolated posterior myocardial infarction: Insights from the national cardiovascular data registry (NCDR). Am Heart J 167: 350-354, 2014.

2. Boersma E, Maas AC, Deckers JW and Simoons ML: Early thrombolytic treatment in acute myocardial infarction: Reappraisal of the golden hour. Lancet 348: 771-775, 1996.

3. Buja LM: Myocardial ischemia and reperfusion injury. Cardiovasc Pathol 14: 170-175, 2005.

4. Guido BC, Zanatelli M, Tavares-de-Lima W, Oliani SM and Damazo AS: Annexin-A1 peptide down-regulates the leukocyte recruitment and up-regulates interleukin-10 release into lung after intestinal ischemia-reperfusion in mice. J Inflamm (Lond) 10: 10,2013.

5. Wang X: Investigational anti-inflammatory agents for the treatment of ischaemic brain injury. Expert Opin Investig Drugs 14: 393-409, 2005.

6. Gong P, Lu Z, Xing J, Wang N and Zhang Y: Traditional Chinese medicine Xuebijing treatment is associated with decreased mortality risk of patients with moderate paraquat poisoning. PLoS One 10: e0123504, 2015.

7. Wang YX, Ji ML, Chen LP, Wu XJ and Wang L: Breviscapine reduces acute lung injury induced by left heart ischemic reperfusion in rats by inhibiting the expression of ICAM-1 and IL-18. Exp Ther Med 6: 1322-1326, 2013.

8. Hou SY, Feng XH, Lin CL and Tan YF: Efficacy of Xuebijing for coagulopathy in patients with sepsis. Saudi Med J 36 $164-169,2015$

9. Xu Q, Liu J, Guo X, Tang Y, Zhou G, Liu Y, Huang Q, Geng Y, Liu Z and Su L: Xuebijing injection reduces organ injuries and improves survival by attenuating inflammatory responses and endothelial injury in heatstroke mice. BMC Complement Altern Med 15: 4, 2015.

10. National Institutes of Health: Guide for the Care and Use of Laboratory Animals. 8th edition. National Academies Press, Washington (DC), 2011.

11. $\mathrm{Yu} \mathrm{P}, \mathrm{Bu} \mathrm{H}$, Wang $\mathrm{H}$, Zhao GP, Zhang $\mathrm{J}$ and Zhou Q: Comparative study on image analysis and manual counting of immunohistochemistry. J Biomed Eng 20: 288-290, 2003 (In Chinese).

12. Zhao Q, Sun C, Xu X, Zhou J, Wu Y, Tian Y, Yuan Z and Liu Z: CD34 ${ }^{+}$cell mobilization and upregulation of myocardial cytokines in a rabbit model of myocardial ischemia. Int J Cardiol 152: 18-23, 2011.

13. Huang CH, Tsai SK, Chiang SC, Wang YY, Chih CL, Weng ZC and Lai ST: Brief pressure overload preconditions rabbit myocardium independent of adenosine receptor activation. Ann Thorac Surg 92: 1727-1732, 2011.

14. Sun ZH: Coronary CT angiography in coronary artery disease: Correlation between virtual intravascular endoscopic appearances and left bifurcation angulation and coronary plaques. Biomed Res Int 2013: 732059, 2013.

15. Arun Prakash, Kailin R. Mesa, Kevin Wilhelmsen, Fengyun Xu, Jeffrey M. Dodd-o, Judith Hellman: Alveolar Macrophages and Toll-like Receptor 4 Mediate Ventilated Lung Ischemia Reperfusion Injury in Mice

16. Hughes SF, Hendricks BD, Edwards DR, Bastawrous SS, Roberts GE and Middleton JF: Mild episodes of tourniquet-induced forearm ischaemia-reperfusion injury results in leukocyte activation and changes in inflammatory and coagulation markers. J Inflamm (Lond) 4: 12, 2007.
17. Ranganathan PV, Jayakumar C, Mohamed R, Dong Z and Ramesh G: Netrin-1 regulates the inflammatory response of neutrophils and macrophages and suppresses ischemic acute kidney injury by inhibiting COX-2 mediated PGE2 production. Kidney Int 83: 1087-1098, 2013.

18. Dziodzio T, Biebl M and Pratschke J: Impact of brain death on ischemia/reperfusion injury in liver transplantation. Curr Opin Organ Transplant 19: 108-114, 2014.

19. Zygner W, Gójska-Zygner O, Bąska P and Długosz E: Increased concentration of serum TNF alpha and its correlations with arterial blood pressure and indices of renal damage in dogs infected with Babesia canis. Parasitol Res 113: 1499-1503, 2014.

20. Lee JW, Kim SC, Ko YS, Lee HY, Cho E, Kim MG, Jo SK, Cho WY and Kim HK: Renoprotective effect of paricalcitol via a modulation of the TLR4-NF- $\kappa$ B pathway in ischemia/reperfusion-induced acute kidney injury. Biochem Biophys Res Commun 444: 121-127, 2014.

21. Guzmán-de la Garza FJ, Ibarra-Hernández JM, Cordero-Pérez P, Villegas-Quintero P, Villarreal-Ovalle CI, Torres-González L, Oliva-Sosa NE, Alarcón-Galván G, Fernández-Garza NE, Muñoz-Espinosa LE, et al: Temporal relationship of serum markers and tissue damage during acute intestinal ischemia/reperfusion. Clinics (Sao Paulo) 68: 1034-1038, 2013.

22. Salim SY, Young PY, Lukowski CM, Madsen KL, Sis B, Churchill TA and Khadaroo RG: VSL\#3 probiotics provide protection against acute intestinal ischaemia/reperfusion injury. Benef Microbes 4: 357-365, 2013.

23. Pérez-de Puig I, Miró F, Salas-Perdomo A, Bonfill-Teixidor E, Ferrer-Ferrer M, Márquez-Kisinousky L and Planas AM: IL-10 deficiency exacerbates the brain inflammatory response to permanent ischemia without preventing resolution of the lesion. J Cereb Blood Flow Metab 33: 1955-1966, 2013.

24. Bodhankar S, Chen Y, Vandenbark AA, Murphy SJ and Offner H: IL-10-producing B-cells limit CNS inflammation and infarct volume in experimental stroke. Metab Brain Dis 28: 375-386, 2013.

25. Volin MV and Koch AE: Interleukin-18: A mediator of inflammation and angiogenesis in rheumatoid arthritis. J Interferon Cytokine Res 31: 745-751, 2011.

26. Celik E, Faridi MH, Kumar V, Deep S, Moy VT and Gupta V: Agonist leukadherin-1 increases CD11b/CD18-dependent adhesion via membrane tethers. Biophys J 105: 2517-2527, 2013.

27. He P, Srikrishna G and Freeze HH: N-glycosylation deficiency reduces ICAM-1 induction and impairs inflammatory response. Glycobiology 24: 392-398, 2014.

28. Pillay J, Kamp VM, Pennings M, Oudijk EJ, Leenen LP, Ulfman LH and Koenderman L: Acute-phase concentrations of soluble fibrinogen inhibit neutrophil adhesion under flow conditions in vitro through interactions with ICAM-1 and MAC-1 (CD11b/CD18). J Thromb Haemos 11: 1172-1182, 2013.

29. Wu R, Zhou Q, Lin S, Ao X, Chen X and Yang J: Effect of cordceps sinensis on the expression of ICAM-1 and VCAM-1 in the kidney of spontaneously hypertensive rats. Zhong Nan Da Xue Xue Bao Yi Xue Ban 35: 152-158, 2010 (In Chinese).

30. Sakai N, Shin T, Schuster R, Blanchard J, Lentsch AB, Johnson WT and Schuschke DA: Marginal copper deficiency increases liver neutrophil accumulation after ischemia/reperfusion in rats. Biol Trace Elem Res 142: 47-54, 2011.

31. Liu MW, Wang YH, Qian CY and Li H: Xuebijing exerts protective effects on lung permeability leakage and lung injury by upregulating Toll-interacting protein expression in rats with sepsis. Int J Mol Med 34: 1492-1504, 2014.

32. Gong P, Lu Z, Xing J, Wang $\mathrm{N}$ and Zhang Y: Traditional Chinese medicine Xuebijing treatment is associated with decreased mortality risk of patients with moderate paraquat poisoning.PLoS One 10: e0123504, 2015. 